\title{
Análisis espacial y temporal de las sequías en el nordeste de Brasil
}

\author{
Paredes, F. J.; H. Barbosa A. y E. Guevara
}

\section{RESUMEN}

El nordeste de Brasil (NEB) es una región afectada por sequías que causan severos impactos sobre los sectores agrícola, hidroeléctrico y de abastecimiento hídrico. Se evaluaron las características más relevantes de las sequías severas acaecidas en el NEB entre 1901-2010 (en lo sucesivo, rachas secas). Se empleó el índice de precipitación estandarizada para medir la anomalía de la precipitación acumulada a escala trimestral (SPI-3). Los patrones subyacentes que configura el SPI-3 en el dominio espacio-temporal, se identificaron aplicando un análisis por componentes principales seguido de un análisis de conglomerados. La periodicidad de las rachas secas se evaluó aplicando un análisis de ondoleta. Entre los resultados, destacan: i) las rachas secas más extensas se ajustan a cuatro patrones espaciales que se intercalan en el tiempo; ii) el área afectada por rachas secas, a escala mensual, tiene un periodo aproximado de 10 meses, mientras que el número de eventos por año muestra periodos de entre 2 y 8,10 y 30 años, respectivamente; iii) las rachas secas en cuanto a su duración, cantidad de eventos por año, recurrencia mensual y área afectada por estos eventos no muestran tendencias de largo plazo, aunque los episodios más extensos inciden con más frecuencia en la temporada húmeda.

Palabras clave: sequía, nordeste de Brasil, variabilidad climática

Paredes, F. J.; H. Barbosa A. and E. Guevara, 2015. Spatial and temporal analysis of droughts in northeastern Brazil. Agriscientia 32 (1): $1-14$

\section{SUMMARY}

Northeast Brazil (NEB) is a region often affected by strong droughts that cause severe impacts over agriculture, hydropower and water supply sectors. The most relevant features of severe droughts that occurred in the entire NEB during the 1901-2010 period (henceforth, dry spells) were evaluated. The standardized precipitation index with a quarterly time scale was used as drought index (SPI3). The underlying patterns that configure the SPI-3 in the spatio-temporal domain were identified applying a principal component analysis followed by a cluster analysis. The dominant periodicities of the dry spells at monthly and 
annual scales were estimated applying a wavelet analysis. The most remarkable results were: i) the longest dry spells are linked to four dominant patterns which are alternated in time; ii) the monthly affected surface by dry spells has a periodicity of approximately 10 months, while the amount of annual dry spells show periods from 2 to 8,10 and $30 \mathrm{yr}$ respectively; iii) the dry spells do not show monotonous trends with relation to its duration, number of events per year and affected area; however, the longest episodes often occur during the main rainy season of the NEB.

Key words: drought, Northeast Brazil, climate variability

F.J. Paredes: UNELLEZ-VIPI / Centro de Investigaciones Hidrológicas y Ambientales de la Universidad de Carabobo (CIHAM-UC), Facultad de Ingeniería, Arquitectura y Tecnología (UNELLEZ-VIPI), 2201, Avenida Universidad, Km. 4, San Carlos, Cojedes, Venezuela. H. Barbosa Alves: Laboratório de Análise e Processamento de Imagens de Satélites (LAPIS), Instituto de Ciências Atmosféricas, Universidade Federal de Alagoas, Maceió, AL - Brasil. E. Guevara: (CIHAM-UC), Universidad de Carabobo, Bárbula, Valencia, Carabobo, Venezuela. Correspondencia a: franklinparedes75@gmail.com

\section{INTRODUCCIÓN}

La sequía es un fenómeno asociado a la variabilidad natural del clima (Botterill \& Cockfield, 2013), que se produce cuando en un lugar determinado la cantidad de precipitación es inferior a la media climatológica de largo plazo durante un periodo prolongado (Organización Meteorológica Mundial, 2005). A diferencia de la aridez, que es una característica permanente, la sequía es un estado transitorio (Allaby, 2003; Ravelo et al., 2014). Cuando esta condición persiste, el aporte pluviométrico puede llegar a ser insuficiente para cubrir las demandas hídricas de un grupo social (Vicente-Serrano et al., 2012). Las sequías se categorizan según su persistencia e impactos sobre la sociedad en cuatro tipos: i) meteorológica: cuando las precipitaciones son menores a la media durante un prolongado periodo de tiempo; ii) agrícola: cuando no hay suficiente humedad en el suelo para el desarrollo de un cultivo en cualquiera de sus fases de crecimiento; iii) hidrológica: cuando los caudales de los ríos no pueden satisfacer los usos establecidos bajo un determinado sistema de gestión de aguas; iv) socioeconómica: cuando la disminución de la disponibilidad de agua ocasiona daños a la población de la zona afectada (Valiente, 2001; Gathara et al., 2006)

Tres parámetros caracterizan las sequías: i) la intensidad, referida al déficit de precipitación; ii) la duración, o tiempo en el cual persiste la condición seca; y iii) la extensión, se refiere al área afectada por la escasez de lluvia (Byun \& Wilhite, 1999). La intensidad suele medirse en términos de la desvia- ción de los aportes respecto a una pauta de precipitación considerada normal (Organización Meteorológica Mundial, 2005). A partir de la intensidad se pueden estimar su duración y extensión superficial (Hayes et al., 1999). Para medir la intensidad de las sequías se han formulado una amplia variedad de índices; así por ejemplo, en Estados Unidos, el índice de sequía de Palmer (1965) fue el primero en ser usado para este propósito (Guttman, 1998), aunque su conceptualización fue mejorada con el pasar de los años (Keyantash \& Dracup, 2002); actualmente, este índice se emplea junto a otros de mayor complejidad en los sistemas de alerta temprana (Organización Meteorológica Mundial, 2005). La selección de un índice de sequía depende en gran medida de la información disponible y del tipo de sequía operacional a monitorear (Byun \& Wilhite, 1999; Barbosa et al., 2015). Hay regiones afectadas por sequías que no tienen suficiente información climática, hidrológica y edáfica. En estos casos, se suele emplear el índice de precipitación estandarizada (SPI, siglas de Standarized Precipitation Index) desarrollado por McKee et al. (1993), cuyo calculo sólo requiere de registros de precipitación mensual (Vicente-Serrano, 2006); en el ámbito internacional, es el más usado (Núñez et al., 2014). Las siglas utilizadas para el índice de precipitación estandarizada provienen del inglés y por ser de uso corriente se han adoptado en el resto de este trabajo.

Las sequías suelen evolucionar muy lentamente, por lo que sus efectos se perciben varias semanas o meses después de su inicio (Organización Meteorológica Mundial, 2005), característica que 
dificulta su predicción (Keyantash \& Dracup, 2002) y explica en gran medida por qué causan grandes pérdidas socioeconómicas en los países que carecen de sistemas de alerta temprana y suficientes recursos económicos para afrontar sus impactos (World Water Assessment Programme, 2009). La ONU (Organización de las Naciones Unidas) ha auspiciado varios esfuerzos internacionales para el control y mitigación de las sequías (Naciones Unidas, 1994). La referencia más importante en esta materia es la Convención para la Lucha contra la Desertificación y las Sequías de la Organización de la Naciones Unidas (Naciones Unidas, 1994), de la cual se han derivado múltiples iniciativas regionales. Según la Comisión Económica para América Latina y el Caribe, el número de episodios secos en América del Sur y el Caribe, durante la década 2000-2009, cuadriplicó el observado en la década 1970-79 (CEPAL, 2010). En Brasil, las sequías meteorológicas e hidrológicas durante la década 2001-10, fueron 2,7 veces mayor que las registradas en el periodo 1991-2001; un aspecto notorio es el hecho de que $60 \%$ de estos eventos ocurrieron en el nordeste de Brasil (en lo sucesivo, NEB) (UFS \& CEPED, 2012) (Figura 1). El NEB es una región de unos $1,6 \times 10^{6} \mathrm{~km}^{2}$ que incluye los estados: Alagoas (AL), Bahia (BA), Ceará (CE), Maranhão (MA), Paraíba (PA), Piauí (PI), Rio Grande do Norte (RN), Sergipe (SE) y Pernambuco (PE). Esta región fue afectada recientemente por una severa sequía que se prolongó desde el año 2012 a 2013 (Blunden \& Arndt, 2014).

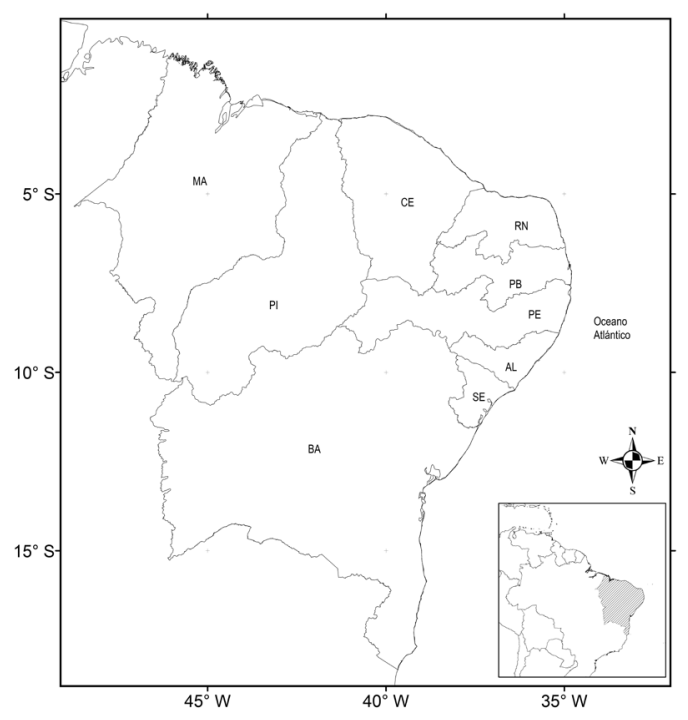

Figura 1. Nordeste de Brasil (zona de estúdio). Se indican los estados: Alagoas (AL), Bahia (BA), Ceará (CE), Maranhão (MA), Paraíba (PA), Piauí (PI), Rio Grande do Norte (RN), Sergipe (SE) y Pernambuco (PE).

La precipitación en el NEB muestra una marcada variabilidad espacial, estacional e interanual (Alves \& Repelli, 1992; Hastenrath \& Greischar, 1993). La precipitación configura un gradiente este-oeste con promedios de 1800 mm/año en la región amazónica de MA, 600 mm/año en el centro del NEB y 1350 mm/año en el litoral (Rao et al., 1993). Según la concentración estacional de las lluvias, se diferencian claramente tres subregiones: i) sur (incluye BA, sur de $\mathrm{MA}$ y $\mathrm{Pl}$ ), en la que las Iluvias ocurren en el periodo NDEF (noviembre, diciembre, enero y febrero) debido a la incidencia de frentes fríos provenientes del hemisferio sur y el incremento de la actividad de la zona de convergencia del Atlántico Sur (ZCAS); ii) norte (agrupa MA, PI, CE y RN; oeste de PA, PE y AL; y el nortenordeste de BA), donde las lluvias se concentran durante EFMA (enero, febrero, marzo y abril) por el paso de la zona de convergencia intertropical (ZCIT), la cual es fuertemente modulada por la temperatura de la superficie del mar en el Atlántico Ecuatorial; iii) este (franja litoral de RN, PA, PE, AL, SE y BA), donde las lluvias se dan en el trimestre AJJ (abril, junio y julio), producto de la interacción entre la ZCIT y los vientos alisios del sudeste, así como por perturbaciones atmosféricas procedentes de África (Kousky, 1979; Molion \& Bernardo, 2002; Kayano \& Andreoli, 2006). Las sequías en el NEB pueden ocurrir en cualquier momento del año; sin embargo, durante el periodo 1990-2000 se observó que fueron más frecuentes en MAM (marzo, abril, mayo), concentrándose en los estados BA, PI, PB y CE (UFS \& CEPED, 2012). Los extremos en la variabilidad interanual de las lluvias en el NEB son atribuidas a: i) la ocurrencia de aguas superficiales anormalmente calientes al norte del Ecuador y frías al sur, durante el trimestre MAM. Este arreglo es conocido como el dipolo del Atlántico, y puede causar un desplazamiento de la ZCIT al norte de su posición normal, al tiempo de modificar la celda de circulación de Hadley e interrumpir, temporalmente, las lluvias en el NEB (Knight et al., 2006; Sutton and Hodson, 2007; Rodrigues et al., 2011); ii) la fase caliente del fenómeno El Niño-Oscilación del Sur (ENOS), durante la cual la celda de circulación de Walker, situada sobre el océano Pacifico ecuatorial, modifica su patrón normal de circulación y origina una fuerte rama zonal descendente que causa una subsidencia a gran escala sobre el NEB, inhibiendo así la nubosidad local (Chaves \& Cavalcanti, 2001; Kayano \& Andreoli, 2004). A escala intra-estacional, los modos más influyentes son: las ondas de Madden-Julian, las cuales favorecen la ocurrencia de veranillos de corta duración (Yamazaki \& Rao, 1977); la dinámica de la ZCAS 
previamente comentada; y los vórtices ciclónicos en altura (Hastenrath, 2000). Otros modos que modulan la variabilidad de las lluvias en el NEB, con una baja frecuencia temporal, son la oscilación del Atlántico Norte (NAO, North Atlantic ocean), la oscilación decadal del Pacifico (PDO, Pacific decadal oscillation) y la oscilación multidecadal del Atlántico (AMO, Atlantic multidecadal oscillation) descritas en detalle en Kayano \& Andreoli (2004) y Kayano \& Capistrano (2014).

Las sequías en el NEB han causado importantes impactos negativos en los sectores agrícola, hidroeléctrico y de abastecimiento hídrico (Livingstone \& Assunção, 1989; Buchmann, 1998; Buckley, 2010). A manera de ejemplo, durante el periodo 1991-2010 afectaron más de $35 \times 10^{6}$ personas; en $\mathrm{PI}$ y BA se registraron, en promedio, 131,59 y 96,80 eventos secos por habitantes $/ \mathrm{km}^{2}$, respectivamente (UFS \& CEPED, 2012). Aunque la variabilidad de las precipitaciones en el NEB y su modulación por parte de los patrones de circulación atmosféricos y oceánicos, de gran escala, como ENOS, ha sido ampliamente documentada (Kousky, 1979; Alves \& Repelli, 1992; Hastenrath \& Greischar, 1993; Rao et al., 1993; Hastenrath, 2000; Chaves \& Cavalcanti, 2001; Kayano \& Andreoli, 2002; Kayano \& Andreoli, 2006; Kayano \& Capistrano, 2014), aún es necesario profundizar el conocimiento sobre este fenómeno, en particular los de mayor intensidad (en lo sucesivo, rachas secas), su evolución en el contexto espacial, así como su periodicidad y persistencia temporal. Estos aspectos constituyen un complemento importante para planificar las acciones de alerta temprana y mitigación por parte de los sectores vulnerables a las sequías en el NEB.

El objetivo de este trabajo fue identificar los patrones espacio-temporales en la ocurrencia de rachas secas y sus principales características en los dominios espacio, tiempo y frecuencia, empleando el SPI como índice de sequía.

\section{MATERIALES Y MÉTODOS}

\section{Zona de estudio}

El estudio se llevó a cabo en el NEB (Figura 1). Las principales actividades económicas de esta región, en el ámbito agropecuario, son la siembra de caña de azúcar y la cría de ganado caprinos (Instituto Brasileiro de Geografia e Estatística, 2006).

\section{Materiales}

La cartografía digital utilizada proviene del Instituto Brasileiro de Geografía y Estadística e incluye los mapas: político-administrativo regional, estatal y municipal del NEB (Instituto Brasileiro de Geografia e Estatística, 2014). Las series mensuales de precipitación fueron suministradas por el Instituto Nacional de Meteorología de Brasil (INMET, 2014) y son parte del Banco de Datos Meteorológicos para la Enseñanza e Investigación (BDMEP, por sus siglas en portugués)

El INMET opera 65 estaciones en el NEB, con series mensuales que van desde el año 1961 al 2013; 19 de estas estaciones tienen $\geq 75 \%$ de años completos con valores mensuales perdidos que varían de 2,83 a 41,35\% concentrados en 1971-72, 1985 y 1990. Por la escasa cobertura espacial y significativa discontinuidad temporal de los registros del INMET, se utilizó la última versión de precipitación mensual reticulada (versión 6) generada por el Centro Global de Precipitación Climatológica (GPCC, Global Precipitation Climatology Center). Este es un producto reticulado, compuesto por celdas de dimensión $0.5^{\circ} \times 0.5^{\circ}$ (latitud-longitud), las cuales cubren totalmente el NEB. A cada celda se asocia una serie mensual de precipitación continua desde 1901 a 2010. El GPCC generó estas series usando registros pluviométricos a escala mundial. La calidad de los registros fue evaluada rigurosamente por el GPCC, mientras que los valores faltantes fueron rellenados empleando el método de interpolación spheremap (Schneider et al., 2014). Para validar el uso de este producto en el NEB, las series pluviométricas del INMET y GPCC, cuyas distancias celda-estación son $\leq 0.707^{\circ}$ (que es el radio interno de una celda, $\sim 78 \mathrm{~km}$ ), fueron contrastadas durante el periodo 1961-2010 (lapso común). Antes de la validación, los registros mensuales del INMET fuera del rango, media $\pm 3 \sigma$, fueron codificados como valores perdidos. Previamente se verificó la normalidad de la sub-series mensual usando una prueba Kolmogorov-Smirnov al $5 \%$ de nivel de confianza; si la referida sub-serie no era normal para este nivel, el registro fue conservado (Kayano \& Andreoli, 2004; Ravelo et al., 2014). Los coeficientes de correlación de Pearson entre las sub-series mensuales INMET-GPCC, con al menos 30 años pareados y estadísticamente significativos de acuerdo a una prueba t-Student ( $\alpha=$ $5 \%$ ), fueron usados como indicador de coherencia (Wilks, 2006). Solo 44 estaciones del INMET cumplieron estos criterios; sus coeficientes de correlación variaron entre 0,313 y 0,965, con un promedio de 0,730 , lo que indica que la variabilidad espacial y temporal de las lluvias en el NEB es capturada apropiadamente. En Brasil, este producto del GPCC fue usado previamente por Paegle \& Mo 
(2002) y Kayano \& Capistrano (2014).

\section{Métodos}

La periodicidad de una serie de tiempo puede estimarse usando una transformada de ondoleta continua (CWT, continuous wavelet transform), que es una técnica de análisis espectral en la que una serie es descompuesta en los dominios tiempo y escala, usando una versión escalada y trasladada de una función base denominada ondoleta madre. A diferencia del análisis espectral de Fourier (Vaseghi, 2000; Bloomfield, 2004), el análisis de ondoletas arroja información simultánea sobre los dominios tiempo y frecuencia de la señal analizada (Addison, 2002). Las sequías configuran un gran número de patrones en el contexto espacial, por lo que es inviable caracterizarles de forma individual. Sin embargo, éstos pueden reducirse a unos pocos patrones, empleando dos técnicas estadísticas, en orden sucesivo: i) un análisis por componentes principales (PCA, principal components analysis) y ii) un análisis de conglomerados (CA, cluster analysis). El PCA crea un grupo de menor tamaño, cuyos miembros, son combinaciones lineales de los patrones mensuales. En general, el PCA agrupa los patrones espaciales observados, de tal forma que los primeros componentes retienen la mayor proporción de la variabilidad original (Chaves \& Cavalcanti, 2001); los componentes resultantes se suelen denominar funciones ortogonales empíricas (EOF, empirical orthogonal function) y desde el punto de vista físico, pueden considerarse como los patrones dominantes (Dommenget \& Latif, 2002). Por otro lado, el CA clasifica los EOFs empleando las puntuaciones como elemento de agrupación; así, resulta un vector ordinal que contiene los patrones espaciales ordenados según su ocurrencia temporal (Wilks, 2006).

La precipitación reticulada sobre el NEB consta de 512 celdas ( $3000 \mathrm{~km}^{2} /$ celda). Cada celda se vincula a una serie mensual continua de precipitación que cubre desde enero 1901 a diciembre 2010 (110 años de periodo analizado). Para minimizar la influencia de posibles valores atípicos, los registros fuera del rango, media $\pm 3 \sigma$, se reemplazaron por el promedio estimado al considerar los vecinos cercanos (según su ubicación, una celda tiene entre uno y ocho celdas adyacentes); 0,55\% de los registros fueron así promediados; la normalidad de cada serie mensual fue chequeada previamente aplicando una prueba Kolmogorov-Smirnov al 5\% de nivel de confianza. Las series resultantes se transformaron al SPI, aplicando el siguiente procedimiento: i) agrupación trimestral móvil; ii) ajuste mensual de cada subserie mensual a una función gamma de dos parámetros (estimados por máxima verosimilitud); iii) cálculo de la probabilidad acumulada de cada elemento, empleando una función gamma incompleta; iv) conversión de cada elemento a un valor normalizado. Este último es el SPI (en lo sucesivo, SPI-3; McKee et al., 1993). Se seleccionó una agrupación trimestral (escala temporal) porque permite detectar eventos secos que modifican la humedad del suelo (Guttman, 1998). A los efectos del estudio, una racha seca ocurre cuando el SPI-3 es clasificado como severamente $\operatorname{seco}(-2.00<\mathrm{SPI}-3 \leq-1.50)$ o extremadamente seco (SPI-3 $\leq-2.00$ ), resultando, por tanto, una variable dicotómica: i) racha seca y ii) racha no seca. En resumen, se crearon dos series de tiempo SPI3: i) natural, sin modificación en los registros; y ii) categorizada, formada por ceros y unos, según la ocurrencia o no de rachas secas: 1 y 0 , respectivamente.

En el contexto espacial, el SPI-3 natural configura 1318 patrones mensuales durante el periodo 1901-2010, los cuales serían excesivamente tediosos de analizar uno a uno; por tanto, estos patrones se redujeron como sigue. Se construyó una matriz de 1318 filas $\times 512$ columnas, que contiene los SPI-3 en cada celda de la retícula por mes. Esta matriz, es normalmente multivariada con un 95\% de confianza, según una prueba de KolmogorovSmirnov (Marsaglia et al., 2003) aplicada a los patrones mensuales. Una prueba de Bartlett (Bartlett, 1951), sobre 100 de estos últimos, escogidos al azar, indicó que están correlacionados con un 95\% de nivel de confianza. Lo anterior sustentó la aplicación del PCA con rotación varimax para identificar los patrones similares (Wilks, 2006). EI PCA generó 10 EOFs inter-ortogonales que retuvieron $76 \%$ de la varianza original (Dommenget \& Latif, 2002). El número de EOFs escogido se basó en la regla de Kaiser (Jolliffe, 2013). El área afectada por un episodio seco severo, durante un mes cualquiera, es proporcional al número de celdas con rachas secas entre el total de celdas que cubren el NEB. En consecuencia, se relacionaron los meses con el porcentaje del NEB afectado por rachas secas, en una serie que se denominó, serie de cobertura seca (en lo sucesivo, SCS). Durante el PCA, los SPI-3 reciben puntuaciones en los EOFs, cuya magnitud refleja la fuerza de la correlación. Puesto que los EOFs se basan en el SPI-3, no pueden discriminar entre la ocurrencia o no ocurrencia de episodios secos. Para solventar esta limitación, las matrices de puntuaciones de los EOFs (1318 filas $x 10$ columnas) y el SCS (1318 filas $x$ una columna) se unieron para crear una nueva matriz. A esta última se le aplicó un CA basado en k-medias usan- 
do el algoritmo de Hartigan-Wong (James et al., 2013), con el objeto de identificar los patrones que tienen, puntuaciones y cobertura seca, similares. En la selección de la cantidad de conglomerados, se consideraron dos criterios-objetivo: suma de cuadrados intergrupal mínima (James et al., 2013) y correlación entre los centros de los conglomerados $\leq 0.36$ (Mo \& Ghil, 1988). Bajo este enfoque, resultaron 11 conglomerados. Un análisis discriminante lineal, como se detalla en Peña et al. (2011), coincidió $90.96 \%$ con la clasificación del CA; además, un análisis de varianza, donde se usó el estadístico lambda de Wilks, indicó que la SCS es discriminada por estos conglomerados con un 99\% de nivel de confianza (Wilks, 2006). Para identificar tendencias monótonas en las series de tiempo se utilizó la prueba de Mann-Kendal, con un nivel de confianza del 95\% (McLeod et al., 1990); el efecto de la correlación serial en esta prueba se minimizó usando el esquema de remuestreo propuesto por Davison \& Hinkley (1997). Una interpolación espacial, basada en el método de la distancia inversa ponderada (IDW), se usó en la generación de los mapas de los conglomerados.

A partir de la SCS se obtuvieron:

i) Grafico simple en el periodo 1901-2010, para observar su evolución temporal (variabilidad interanual).

ii) Grafico de caja-bigote agrupado por meses, para caracterizar su variabilidad intermensual y los meses donde las rachas secas tienen mayor extensión.

iii) Espectro de potencia de ondoleta (WPS, wavelet power spectrum), para caracterizar la variable SCS en el dominio tiempo-frecuencia. EI WPS requiere que la serie analizada sea estacionaria (Addison, 2002); por tanto, previo al análisis, a cada registro de la SCS se le restó la media, luego la diferencia resultante fue normalizada por la desviación estándar muestral, seguido se determinó y restó la tendencia lineal. El sesgo en el WPS se corrigió con la técnica descrita por Liu et al. (2007). El procedimiento computacional para generar el WPS, así como los criterios que sustentan la selección de la ondoleta madre Morlet, se detallan en Torrence \& Compo (1998) y Torrence \& Webster (1999).

iv) WPS de la serie de tiempo, número total de meses con rachas secas por año, con el objeto de identificar periodicidades subyacentes en esta variable; esta última resulta de totalizar los meses, por año, con cobertura mayor a cero, en la SCS. En lo sucesivo, la expresión "racha seca de gran cobertura" hace referencia a un evento seco mensual, que consigue afectar una superficie $\geq 25 \%$ del NEB.

\section{RESULTADOS Y DISCUSIÓN}

\section{Patrones SPI-3 mensuales relacionados con las rachas secas en el NEB}

El SPI-3 sobre el NEB durante el periodo 19012010, conforma 11 patrones espaciales, los cuales explican $76 \%$ de la varianza original del SPI-3. Cuatro de esos patrones (en lo sucesivo, tipos) se asocian con la ocurrencia de rachas secas de gran cobertura (Figura 2). Sus principales características son:

Tipo 1: es el de mayor severidad. Afecta en promedio $81,21 \%$ del NEB (Figura 2a). Cubre una extensa región en la franja costera de los estados BA, SE, AL, PB, PE, RN y CE, y gran parte de las tierras del interior de MA, BA y PI. Este tipo está conformado, en gran medida, por rachas extremadamente secas. Es un patrón excepcional, pues sólo se observó en 1903 y 1909 (Figura 4a), con mayor frecuencia en el mes de julio (Figura 3a).

Tipo 2: incide sobre el $51,53 \%$ de la superficie del NEB. A diferencia del patrón tipo 1, las rachas severamente secas son dominantes, las cuales se extienden sobre la porción continental de los estados BA, PE, PB y RN, y casi totalmente en CE, PI y MA (Figura 2b). Su modo de ocurrencia a lo largo del año sugiere que no sigue un patrón estacional (Figura 3b). Este patrón tipo fue dominante en el sub-periodo 1901-20 y, en menor grado, 1950-60 (Figura 4b). Los episodios más extensos (cobertura $\geq 50 \%$ del NEB) se concentran en Feb-Mar.

Tipo 3: en promedio, afecta $34,63 \%$ del NEB. Es dominado por rachas moderadamente secas (Figura 2c). Este patrón se da en cualquier mes del año, pero su máxima extensión ( $\geq 38 \%$ del NEB) ocurre durante Jul-Ago (Figura 3c). Su desarrollo en el contexto temporal (Figura 4c) muestra cierta similitud con el observado en el tipo 2 (Figura 4b).

Tipo 4: en promedio, actúa sobre $25,12 \%$ del NEB. Es dominado por rachas moderadamente secas. Estas últimas estructuran dos núcleos bien definidos, centrados en el interior de MA y en la triple frontera de los estados BA, PE y PI (Figura 2d). La evidencia observacional indica que puede ocurrir en cualquier mes (Figura 3d). Su pauta de ocurrencia en el dominio tiempo (Figura 4d) guarda cierta semejanza con el patrón tipo 3 (Figura 4c). Es un patrón bastante común, en particular durante los sub-periodos 1901-20, 1980-90 y 2005-10 

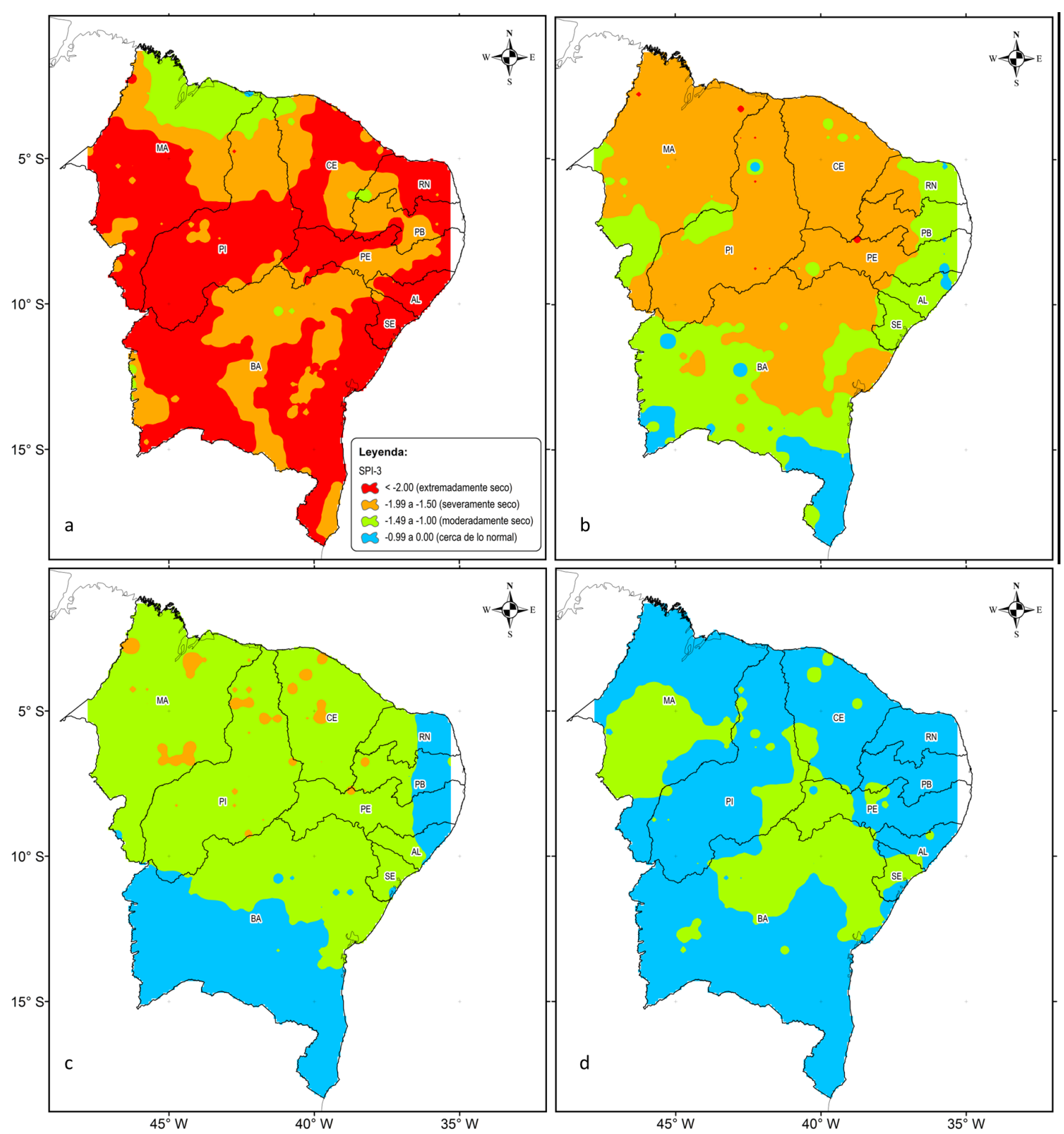

Figura 2. Patrones mensuales SPI-3 promedios vinculados con la ocurrencia de rachas seca de gran cobertura durante el periodo 1901-2010. La cantidad de área afectada por las rachas secas disminuye de a) hasta d), y se les denomina en este orden, patrones tipo 1, 2, 3 y 4, respectivamente.

(Figura 4d).

Los patrones tipos mostrados en la Figura 2 sugieren, a priori, una secuencia mensual de ocurrencia: tipo 4 - tipo 3 - tipo 2 - tipo 1; sin embargo, el cálculo de las probabilidades de transición arrojó los siguientes resultados: 8,51\% del tipo 4 a tipo 3; 4,00\% del tipo 3 a tipo 2; y 0,00\% del tipo 2 a tipo 1 (Tabla 1), lo que indica que esta secuencia no es la ruta de evolución dominante de las rachas secas. Otros indicadores relevantes estimados fueron la persistencia temporal, con $14,89 \%$ del tipo 4 a tipo $4,0,00 \%$ del tipo 3 a tipo 3, 36,00\% del tipo 2 a tipo 2 y $60,00 \%$ del tipo 1 a tipo 1; y la transición hacia patrones con poca cobertura seca $(\leq 25 \%$ del NEB, en lo sucesivo se denominan, genéricamente, tipo 0 ), con $60 \%$ del tipo 4 a tipo 0, 83\% del tipo 3 a tipo 0, 20\% del tipo 2 a tipo 0 y $0 \%$ del tipo 1 a tipo 0 . En resumen, los patrones tipos 1 y 2 tienden a ser persistentes, mientras que los tipos 4 y 3 suelen evolucionar a 

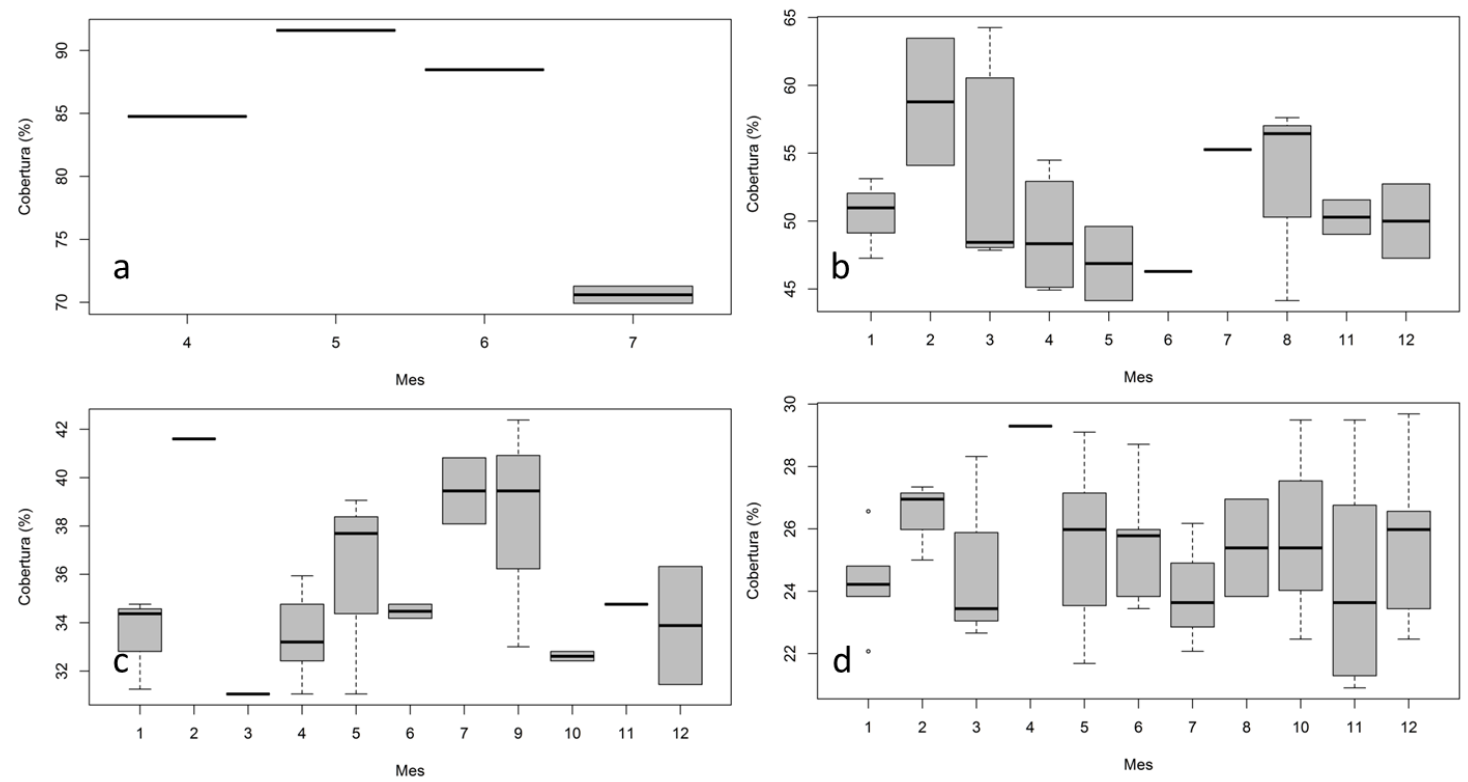

Figura 3. Distribución mensual y rango de cobertura sobre el NEB de los episodios secos que agrupan los patrones de la Figura 2 y 4. Las letras muestran la correspondencia entre las figuras.
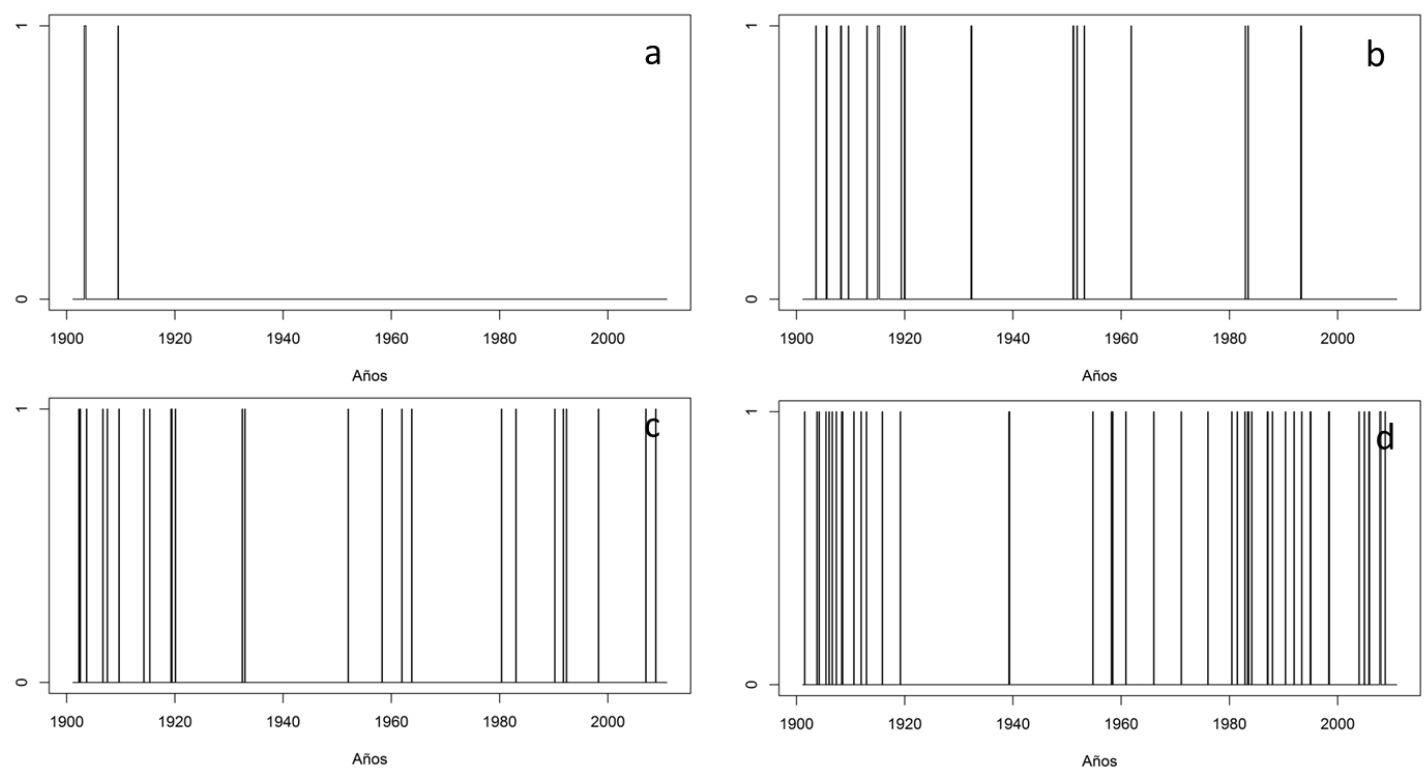

Figura 3. Secuencia mensual de ocurrencia de los patrones mostrados en la Figura 2 durante el periodo 1901-2010. Las letras muestran la correspondencia entre las figuras. Se categorizan en: Ocurrencia $=1$, No ocurrencia $=0$.

patrones con poca cobertura seca (tipo 0). Hay que hacer notar que los patrones tipo 0, pueden agrupar rachas extremadamente secas, pero a diferencia de los tipos 1 al 4, estos, independientemente del patrón que adopten en el contexto espacial (extendida como tipo 1, o nucleada como tipo 4), no logran incidir más del 25\% del NEB. Es muy probable que los fenómenos ENOS y el dipolo del Atlántico, por ser los principales moduladores de la variabilidad climática en el NEB (Molion \& Bernardo, 2002), controlen estas transiciones por vía de una modificación temporal de los patrones 
Tabla 1. Probabilidad de transición entre los patrones SPI-3 mensuales promedios

\begin{tabular}{|c|c|c|c|c|c|c|c|c|c|c|c|}
\hline \multirow[b]{2}{*}{ Desde... } & \multicolumn{11}{|c|}{ A... } \\
\hline & P1 & P2 & P3 & P4 & P5 & P6 & P7 & P8 & P9 & P10 & P11 \\
\hline P1 & 36,00 & 32,00 & 0,00 & 0,00 & 0,00 & 0,00 & 12,00 & 4,00 & 4,00 & 12,00 & 0,00 \\
\hline P2 & 4,00 & 0,00 & 4,00 & 4,00 & 0,00 & 0,00 & 28,00 & 28,00 & 20,00 & 12,00 & 0,00 \\
\hline P3 & 0,00 & 1,21 & 54,84 & 11,69 & 0,00 & 10,89 & 0,81 & 6,05 & 2,02 & 0,00 & 12,50 \\
\hline P4 & 0,00 & 0,60 & 17,86 & 54,17 & 0,00 & 6,55 & 1,79 & 4,76 & 1,79 & 1,19 & 11,31 \\
\hline P5 & 40,00 & 0,00 & 0,00 & 0,00 & 60,00 & 0,00 & 0,00 & 0,00 & 0,00 & 0,00 & 0,00 \\
\hline P6 & 0,00 & 0,00 & 16,58 & 5,53 & 0,00 & 49,75 & 1,01 & 3,52 & 3,52 & 1,51 & 18,59 \\
\hline P7 & 7,46 & 5,97 & 4,48 & 0,00 & 0,00 & 5,97 & 11,94 & 14,93 & 16,42 & 10,45 & 22,39 \\
\hline P8 & 2,26 & 1,50 & 7,52 & 5,26 & 0,75 & 11,28 & 8,27 & 18,80 & 12,78 & 5,26 & 26,32 \\
\hline P9 & 1,28 & 2,56 & 6,41 & 8,97 & 1,28 & 6,41 & 11,54 & 25,64 & 14,10 & 7,69 & 14,10 \\
\hline P10 & 8,51 & 8,51 & 6,38 & 0,00 & 0,00 & 8,51 & 21,28 & 14,89 & 8,51 & 14,89 & 8,51 \\
\hline P11 & 0,00 & 0,50 & 13,57 & 11,06 & 0,00 & 17,09 & 6,03 & 16,58 & 7,04 & 4,52 & 23,62 \\
\hline
\end{tabular}

Nota: P5, P1, P2 y P10 corresponden los patrones tipos 1, 2, 3 y 4 mostrados en la Figura 2a, 2b, 2c y 2d, respectivamente. En los patrones restantes, las rachas secas, en promedio, cubren $\leq 25 \%$ de la superficie del NEB y genéricamente, se les denomina, patrones tipo 0. A manera de ejemplo, la probabilidad de transición mensual desde el patrón P7 al patrón P2 es 5,97\%

de circulación atmosféricos en el NEB (Hastenrath, 2000; Chaves \& Cavalcanti, 2001) y/o la posición de la ZCIT en el Océano Atlántico (Sutton and Hodson, 2007); sin embargo, la evaluación de esta hipotética causalidad está fuera del alcance del artículo.

\section{Características en el dominio tiempo-frecuencia de las rachas secas severas en el NEB}

Las rachas seca de gran cobertura, según se observa en la Figura 5, ocurrieron en: 1903 (7 meses); 1908 y 1915 (5 meses); 1919 y 1932 (4 meses); y 1902, 1905, 1909, 1951, 1993, 1998 y 2007 (3 meses). Estos eventos son más frecuentes durante abril-mayo-junio (Figura 6), y persisten de uno a siete meses (duración): la más larga se registró en 1903 (abril-octubre); la más extensa, en mayo de 1903 (92\% del NEB).

La incidencia de las rachas secas a escala anual (cantidad de eventos por año) muestra una fuerte periodicidad en las frecuencias, que van desde 2 hasta 8 años, y en menor grado, 10 y 30 años (Figura 7b). Estos valores coinciden bastante bien con las frecuencias de algunos modos que juegan un importante rol en la modulación de la variabilidad interanual de las lluvias en el NEB. El primer rango, por ejemplo, es coherente con la actividad de ENOS (Chaves \& Cavalcanti, 2001; Kayano \& Andreoli, 2006), el cual evoluciona de forma cuasicíclica a intervalos que varían entre 2 y 8 años (Torrence \& Compo, 1998); en segundo lugar, la señal de 10 años es similar a la observada en la PDO, que a escala decadal puede afectar las pautas pluviométricas en el NEB (Kayano \& Capistrano, 2004); finalmente, la oscilación de 30 años es análoga a la $\mathrm{AMO}$, la cual se sabe que modula la persistencia de las fases del ENOS a muy baja frecuencia (Kayano \& Capistrano, 2014). Por otro lado, el área mensual afectada por las rachas secas también muestra una periodicidad subyacente, sólo que en este caso es próxima a 10 meses (pico absoluto en la Figura 8b), y discontinua en el dominio tiempo (Figura 8a). Esto último sugiere que los episodios secos, a escala mensual, evolucionan formando picos de cobertura cada 10 meses; es decir, muestran una expansión del área afectada seguida por su retraimiento gradual, repitiéndose este proceso hasta el fin del episodio seco. Sin embargo, esta cualidad sólo es evidente en los periodos 1901-16, 1919-21, 1933-34, 1952-53 y 1954-55, por lo que se infiere que es una condición inherente de las rachas secas de gran cobertura (Figura 5). Un aspecto de importancia que revela el contraste de las Figuras 5 y 7 , es el hecho de que la cantidad de meses afectados por rachas secas durante un año, independientemente de su extensión superficial, no es proporcional a la incidencia de rachas secas de gran cobertura; al contrario, estas últimas, según los resultados de Torrence \& Compo (1998), son atribuibles en gran medida, a la ocurrencia de la fase caliente del ENOS (El Niño) en coexistencia con la fase fría de la AMO (Kayano \& Capistrano, 2014).

En el periodo 1901-2010 se notó que la cantidad de rachas secas de gran cobertura por año, 


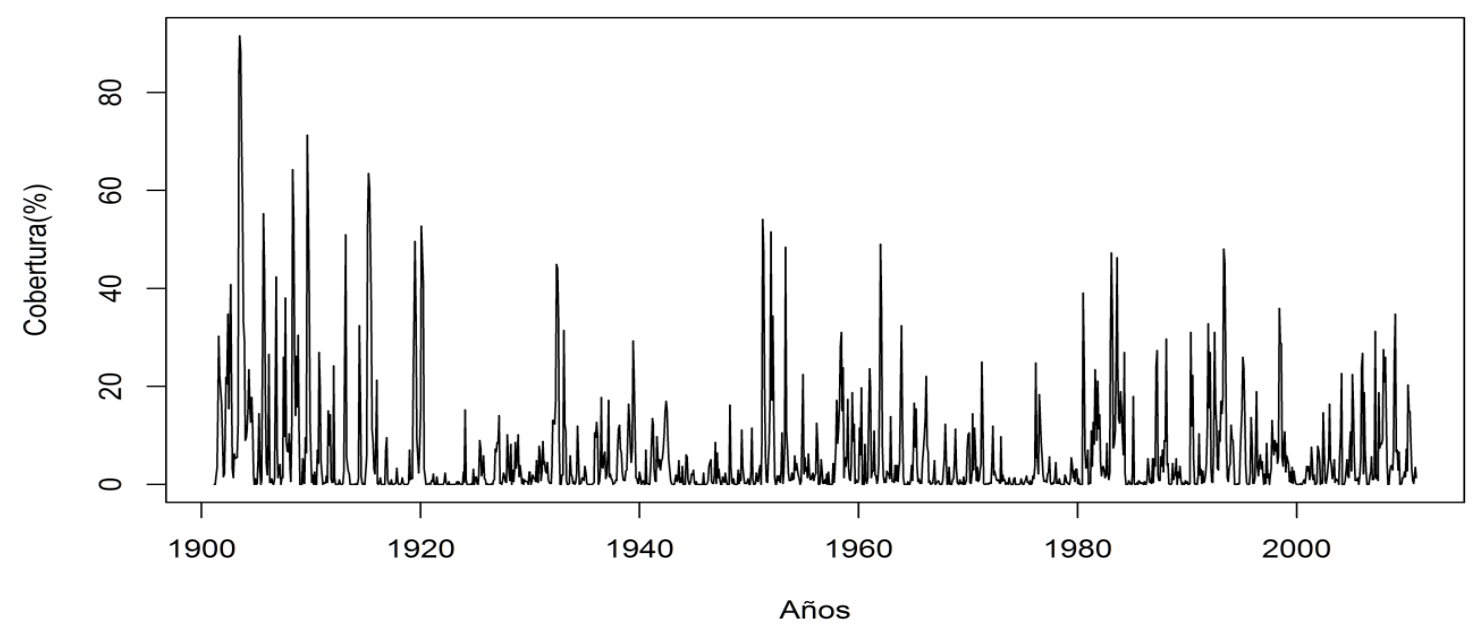

Figura 5. Porcentaje del NEB afectado por rachas secas durante el periodo 1901-2010.

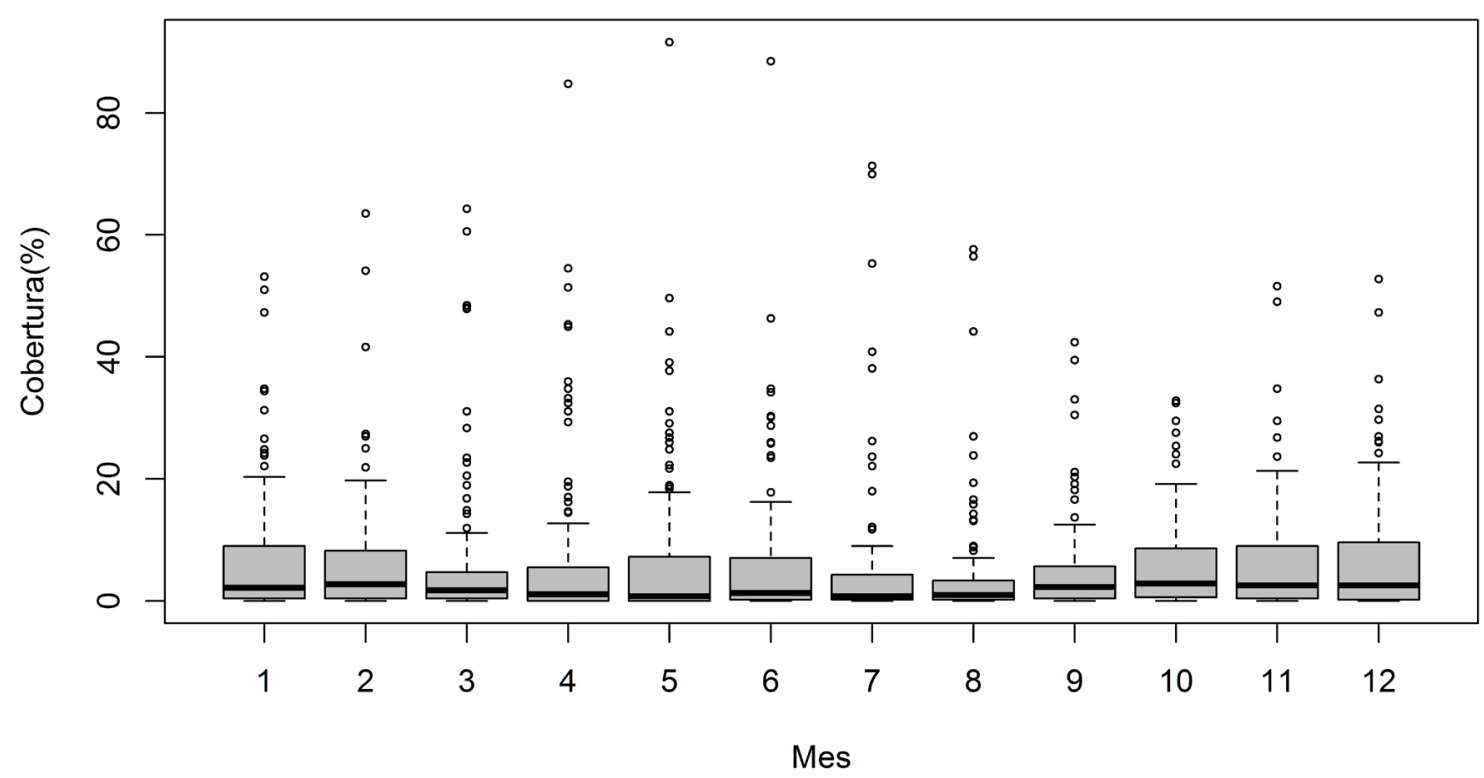

Figura 6. Igual a la Figura 5, pero clasificado por mes. En cada caja, la banda negra representa la mediana, los extremos son los percentiles 25 y 75 (rango intercuartilico) y los círculos son observaciones situadas fuera de $\pm 1,5$ veces el rango intercuartilico.

así como sus duraciones (expresadas en meses), han disminuido; en contraste, el intervalo de tiempo sucesivo entre estos eventos y su incidencia en el bimestre Sep-Oct aumentaron, aunque estos cambios no son estadísticamente significativos. Es posible que exista una conexión entre estas sutiles variaciones y una tendencia positiva de largo plazo observada en las lluvias del NEB (Marengo, 1998), que ha sido atribuida a un atípico desplazamiento de la ZCIT más al sur de su posición nor- mal (Rao et al., 1993). De hecho, algunos estudios han sugerido que el incremento de la temperatura superficial del mar en la banda $20-30^{\circ} \mathrm{S}$ sobre el Océano Atlántico, durante el verano, puede causar tal anomalía (Chaves \& Cavalcanti, 2001; Kayano \& Andreoli, 2004; Kayano \& Capistrano, 2014).

Las rachas secas de gran cobertura son eventos aperiódicos de moderada a baja recurrencia (Figura 5 y 8), pero frecuentes en Abr-May-Jun y Dic (Figura 6). Esto explica la escasez o falta total 

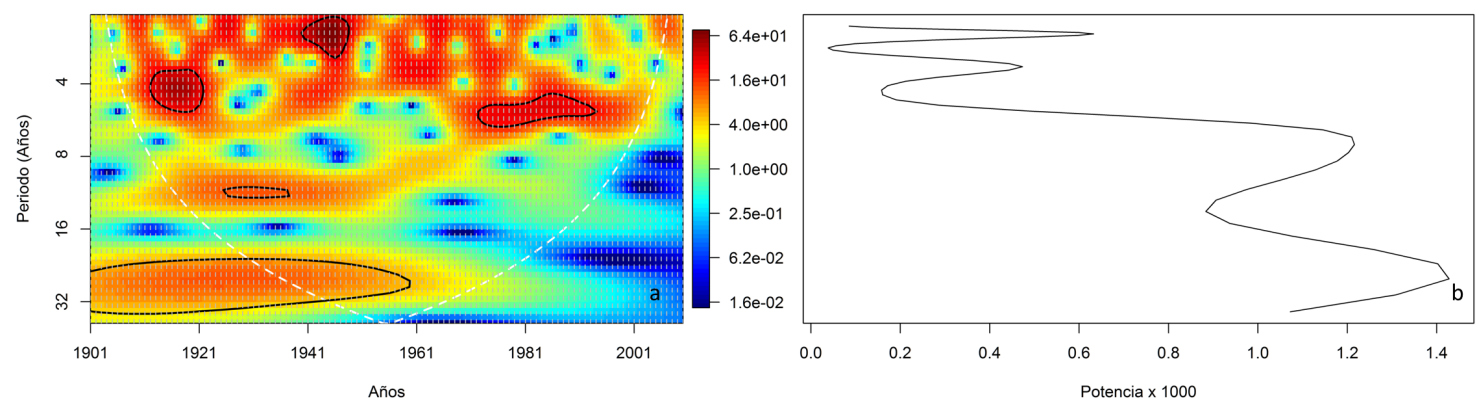

Figura 7. Espectro de ondeleta de la serie de tiempo, número total de meses con rachas secas por año, después de haber sido normalizada y removida su tendencia lineal: a) espectro de potencia local; b) espectro de potencia global. La banda de color representa la energía de la señal en el espectro de potencia local (adimensional). La línea segmentada de color blanco en el espectro de potencia local simboliza el cono de influencia. Las regiones encerradas en el espectro de potencia local, por las líneas gruesas de color negro, son diferentes de un proceso de ruido rojo según una prueba chi-cuadrada a un nivel de confianza del 95\%.
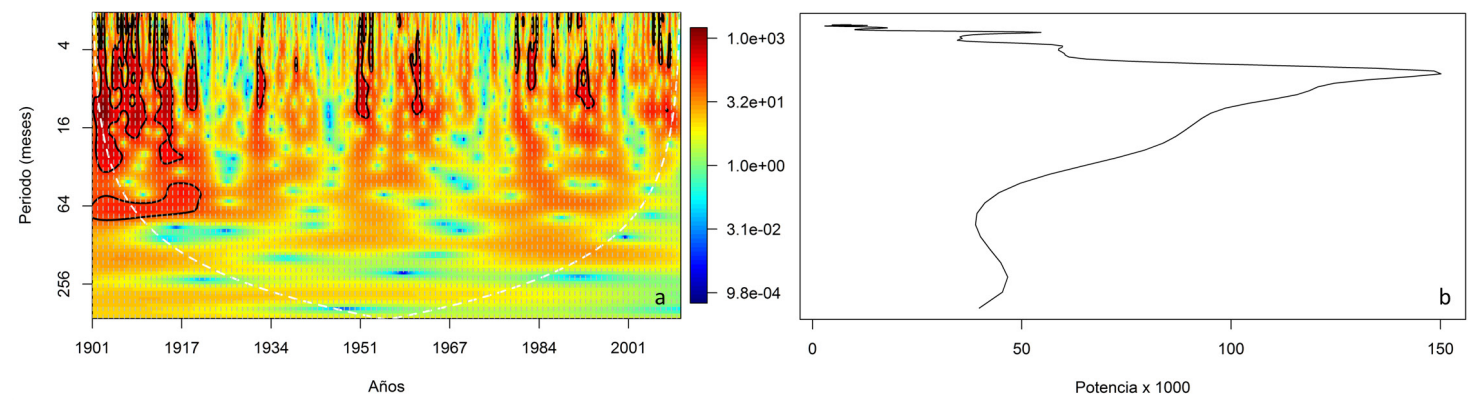

Figura 8. Igual a la Figura 7, pero se representa la serie de tiempo cobertura seca mensual (SCS).

de lluvias que suele observarse durante la estación húmeda de las subregiones norte y sur (estados $\mathrm{BA}, \mathrm{PI}, \mathrm{PB}$ y CE) del NEB, así como la alta incidencia de estos eventos durante el periodo 1990-2000, previamente reportada por UFS \& CEPED (2012). La escasez de lluvias causa un complejo encadenamiento de impactos socioeconómicos negativos sobre los sectores agrícola, hidroeléctrico y de abastecimiento hídrico (Livingstone \& Assunção, 1989; Buchmann, 1998; Buckley, 2010), aunque la compilación documental realizada por Carvalho (2012) sugiere que tales episodios han disminuido su ocurrencia desde el siglo XVIII en el NEB. Un aspecto relevante de este fenómeno es el hecho de que los episodios más severos pueden simplificarse eficientemente a unos pocos patrones espaciales (Figura 2), que se suceden entre sí en el contexto temporal (Figuras 3 y 4 ). Sin embargo, es necesario puntualizar que, por ahora, los factores que modulan la transición entre dichos patrones son desconocidos, aunque los resultados señalan que es muy probable que el ENOS y el dipolo del Atlántico jueguen un rol importante. Por otro lado, no hay evidencia observacional que advierta un cambio en la recurrencia, la evolución o la persis- tencia de las rachas secas en el NEB. Ante este contexto, sólo queda indicar que las actividades económicas dependientes de la ocurrencia de lluvias, en particular el sector agrícola, deben desarrollar e implementar estrategias de adaptación a estos eventos, pues queda claro que son parte de la variabilidad natural del clima en el NEB. El riesgo a las sequías meteorológicas, bajo la concepción clásica de riesgo, no es uniforme en el NEB (Figura 2), porque hay subregiones donde esta amenaza climática es marcadamente recurrente.

\section{CONCLUSIONES}

Un análisis por componentes principales, seguido por uno de conglomerados, permitió identificar los patrones espaciales mensuales dominantes que estructura el SPI-3 sobre el NEB, y que, además, están relacionados con la ocurrencia de sequías locales severamente y extremadamente secas (rachas secas), que afectan más del 25\% del NEB. También, en un contexto probabilístico, se determinó la manera cómo evolucionan las rachas secas y sus características predominantes en 
el dominio tiempo-frecuencia, usando un análisis de ondoleta.

Las rachas secas en el NEB muestran una alta variabilidad estacional e interanual; sin embargo, los eventos más extensos se ajustan a cuatro patrones espaciales que se intercalan entre sí, en el dominio tiempo. La alternancia de estos patrones posiblemente sea modulada por la interacción que se da entre los modos ENOS, PDO y AMO. Por otro lado, las rachas secas, a escala mensual, no muestran tendencias monótonas de largo plazo en cuanto a intensidad, cobertura espacial ni meses de mayor incidencia; por tanto, los eventos observados en el periodo 1901-2010 son atribuibles a la variabilidad natural del clima en el NEB.

Si bien las condiciones secas en el NEB, especialmente durante la última década (UFS \& CEPED, 2012) y, en particular, en la reciente sequía 201213 (Blunden \& Arndt, 2014), han afectado en gran magnitud a los sectores económicos y sociales, la evidencia observacional indica con bastante certeza que la región no está ante un cambio atípico en las condiciones climáticas, sino que, más bien, los sectores económicos vulnerables a estos eventos (en especial, el agrícola) probablemente no han desarrollado políticas adecuadas de adaptación a la variabilidad climática del entorno donde llevan a cabo sus actividades.

\section{AGRADECIMIENTOS}

Los autores agradecen al Instituto de Meteorología de Brasil (INMET) por proveer la información pluviométrica. Este estudio fue financiado por las agencias brasileñas CAPES/CEMADEN/MTCI bajo el proyecto Análise e Previsão dos Fenômenos Hidrometeorológicos Intensos do Leste do Nordeste Brasileiro: Convocatoria Conjunta - Aviso Pró-Alertas en 24/2014.

\section{BIBLIOGRAFÍA}

Addison, P.S., 2002. The Illustrated Wavelet Transform Handbook. CRC Press, London, 368 pp.

Allaby, M, 2003. Dangerous Weather: Droughts. Facts On File, Inc, New York, USA, 225 pp.

Alves, J.M. and S.A. Repelli, 1992. A variabilidade pluviométrica no setor norte do nordeste e os eventos El Niño-Oscilação Sul (ENOS). Revista Brasileira de Meteorologia 7: 583-592.

Barbosa, H.A.; T.V. Lakshmi and L.R. Silva, 2015. Recent trends in vegetation dynamics in the South America and their relationship to rainfall. Natural Hazards (Dor- drecht. Online) 75: 1-17

Bartlett, M., 1951. The Effect of Standardization on a chi square: Approximation in Factor Analysis. Biometrika 38: 337-344.

Bloomfield, P., 2004. Fourier analysis of time series: An Introduction. John Wiley \& Sons, USA, 288 pp.

Blunden, J. and D.S. Arndt, 2014. State of the Climate in 2013. Bulletin of American Meteorological Society 95(7): S1-S257.

Botterill, L.C. and G. Cockfield, 2013. Science, Policy, and Wicked Problems. In Righetti, Botterill L. and G. Cockfield (Eds.): Drought, Risk Management, and Policy: Decision Making under Uncertainty. CRC Press, pp. 1-16.

Buchmann, J., 1998. Aspectos humanos, geográficos e meteorológicos do Nordeste Brasileiro. Anuário do Instituto de Geociências 21: 21-26.

Buckley, E., 2010. Drought in the sertão as a natural or social phenomenon: establishing the Inspetoria Federal de Obras Contra as Secas, 1909-1923. Boletim do Museu Paraense Emílio Goeldi. Ciências Humanas 5: 379-398.

Byun, H.R. and D.A., Wilhite, 1999. Objective Quantification of Drought Severity and Duration. Journal of Climate 12: 2747-2756.

Carvalho, O., 2012. As secas e seus impactos. In: A questão da água no Nordeste. Agência Nacional de Águas - Ministério do Meio Ambiente, Brasília, Brasil, pp. 45-99.

Centro Global de Precipitación Climatológica, 2014. Precipitación mensual reticulada global, versión 6. < http:// climexp.knmi.nl/data/> Consultada el 15/02/2014

CEPAL (Comisión Económica para América Latina y el Caribe), 2010. Gráficos Vitales del Cambio Climático para América Latina y El Caribe. CEPAL, edición especial para la CP16/CP-RP 6, Santiago, Chile, 44 pp.

Chaves, R. and I. Cavalcanti, 2001. Atmospheric circulation features associated with rainfall variability over Southern Northeast Brazil. Monthly Weather Review 129: 2614- 2626.

Davison, A.C. and D.V. Hinkley, 1997 Bootstrap Methods and Their Application. Cambridge University Press, UK, London, $582 \mathrm{pp}$.

Dommenget, D. and M., Latif, 2002. A Cautionary Note on the Interpretation of EOFs. Journal of Climate 15: 216- 225

Gathara, S.T.; L.G. Gringof, E. Mersha, K.C. Sinha and P. Spasov, 2006. Impacts of desertification and drought and other extreme meteorological events. WMO/TD No. 1343, Geneva, Switzerland, 88 pp.

Guttman, N., 1998. Comparing the Palmer Drought Index and the Standardized Precipitation Index. Journal of 
the American Water Resources Association 34 (1): 113-121.

Hastenrath, S. and L. Greischar, 1993. Circulation mechanisms related to Northeast Brazil rainfall anomalies. Journal of Geophysical Research 98: 5093-5102.

Hastenrath, S., 2000. Interannual and longer-term variability of upper air circulation in the northeast Braziltropical Atlantic sector. Journal of Geophysical Research 105: 7327-7335.

Hayes, M.J.; M. Svoboda, D.A. Wilhite and O. Vanyarkho, 1999. Monitoring the 1996 Drought Using the Standardized Precipitation Index. Bulletin of the American Meteorological Society 80(3):429-438.

Instituto Brasileiro de Geografia e Estatística, 2006. Censo Agropecuário 2006. Ministério do Planejamento, Orçamento e Gestão. Instituto Brasileiro de Geografia e Estatística. Rio de Janeiro, 141 pp.

Instituto Brasileiro de Geografia e Estatística, 2014. Produtos de Geociências. < http://downloads.ibge.gov. br/> Consultada el 12/10/2014.

INMET (Instituto Nacional de Meteorología de Brasil), 2014. Banco de Datos Meteorológicos para la Enseñanza e Investigación. <http://www.inmet.gov.br/ portal/> Consultada el 12/10/2014

James, G.; D. Witten, T. Hastie and R. Tibshirani, 2013. An introduction to statistical learning. Springer, $426 \mathrm{pp}$.

Jolliffe, I., 2013. Principal Component Analysis. Springer Science \& Business Media, $271 \mathrm{pp}$.

Kayano, M.T. and R.V. Andreoli, 2002. Clima da Região Nordeste do Brasil. En Cavalcanti, N. J. (Eds.): Tempo e Clima no Brasil. 1 ed. São Paulo: Oficina de Textos, pp. 212-233.

Kayano, M.T. and R.V. Andreoli, 2004. Decadal variability of northern northeast Brazil rainfall and its relation to tropical sea surface temperature and global sea level pressure anomalies. Journal of Geophysical Research 109: C11011.

Kayano, M.T. and R.V. Andreoli, 2006. Relationships between rainfall anomalies over northeastern Brazil and the El Niño-Southern Oscillation. Journal of Geophysical Research 111: D13102

Kayano, M.T. and V.P. Capistrano, 2014. How the Atlantic Multidecadal Oscillation (AMO) modifies the ENSO influence on the South American rainfall. International Journal of Climatology 34: 162-178.

Keyantash, J. and J. A. Dracup, 2002. The quantification of drought: an evaluation of drought indices. Bulletin of the American Meteorological Society 83(8): 11671180.

Knight, J.R.; C.K. Folland and A.A. Scaife, 2006. Climate impacts of the Atlantic multidecadal oscillation. Geophysical Research Letters 33: L17706
Kousky, V.E., 1979. Frontal influences on Northeast Brazil. Monthly Weather Review 107: 1140- 1153.

Liu, Y.; X. San Liang and R. H. Weisberg, 2007. Rectification of the Bias in the Wavelet Power Spectrum. Journal of Atmospheric and Oceanic Technology 24:20932102.

Livingstone, I. and M. Assunção, 1989. Government Policies towards Drought and Development in the Brazilian Sertão. En SAGE (Eds.): Development and Change. London, Newbury Park and New Delhi, pp. 461-500.

Marengo, J. A.; J. Tomasella and C. Uvo, 1998. Trends in streamflow and rainfall in tropical South America: Amazonia, eastern Brazil, and northwestern Peru. Journal of Geophysical Research 103: 1775-1783.

Marsaglia, G; W. Tsang and J. Wang, 2003. Evaluating Kolmogorov's distribution. Journal of Statistical Software 8: 1-4.

McKee, T.B.; N.J. Doesken and J. Kleist, 1993. The relationship of drought frequency and duration to time scales. Preprints, Eighth Conference on Applied Climatology, Anaheim, California, American Meteorology Society, pp. 179-184.

McLeod, A.; K. Hipel and B. Bodo, 1990. Trend analysis methodology for water quality time series. Environmetrics 2: 169-200.

Mo, K. and M. Ghil, 1988. Cluster analysis of multiple planetary flow regimes. Journal of Geophysical Research 93: 10927-10952.

Molion, L.C. and S.O. Bernardo, 2002. Uma revisão da dinâmica das chuvas no Nordeste Brasileiro. Revista Brasileira de Meteorologia 17: 1-10.

Naciones Unidas, 1994. Convención de las Naciones Unidas de Lucha contra la Desertificación en los Países Afectados por Sequía Grave o Desertificación, en particular en África. Naciones Unidas, Paris, London, $66 \mathrm{pp}$.

Nuñez, J.; D. Rivera, R. Oyarzún and J.L. Arumí, 2014. On the use of Standardized Drought Indices under decadal climate variability: Critical assessment and drought policy implications. Journal of Hydrology 517: 458-470.

Organización Meteorológica Mundial, 2005. Vigilancia y alerta temprana de la sequía: conceptos, progresos y desafíos futuros. OMM-N 1006 , Ginebra, Suiza, 28 pp.

Paegle, J. and K. Mo, 2002. Linkages between summer rainfall variability over South America and sea surface temperature anomalies. Journal of Climate 12: 13891407.

Palmer, W.C., 1965. Meteorological drought. U.S. Department of Commerce Weather Bureau Research Paper 45, $58 \mathrm{pp}$.

Peña, J. C.; M. Aran, J. Cunillera and J. Amaro, 2011. At- 
mospheric circulation patterns associated with strong wind events in Catalonia. Natural Hazards and Earth System Sciences 11: 145-155

Rao, V.; M. De Lima and S. Franchito, 1993. Seasonal and interannual variations of rainfall over Eastern Northeast Brazil. Journal of Climate 6: 1754- 1763.

Ravelo, A.C.; R. Ramos y J.C. Douriet, 2014. Detección, evaluación y pronóstico de las sequías en la región del Organismo de Cuenca Pacífico Norte, México. Agriscientia 31(1):1-14.

Rodrigues, R.; R. Haarsma, E. Campos and T. Ambrizzi, 2011. The impacts of Inter-El Niño variability on the Tropical Atlantic and Northeast Brazil climate. Journal of Climate 24: 3402-3422.

Schneider, U.; A. Becker, P. Finger, A. Meyer-Christoffer, M. Ziese and B. Rudolf, 2014. GPCC's new land surface precipitation climatology based on quality-controlled in situ data and its role in quantifying the global water cycle. Theoretical and Applied Climatology 115: $15-40$.

Sutton, R.T. and D.L. Hodson, 2007. Climate response to basin-scale warming and cooling of the North Atlantic Ocean. Journal of Climate 20: 891-907.

Torrence, Ch. and G. Compo, 1998. A Practical Guide to Wavelet Analysis. Bulletin of the American Meteorological Society 79(1): 61-78.

Torrence, Ch. and P. Webster, 1999. Interdecadal Changes in the ENSO-Monsoon System. Journal of Climate 12: 2679-2690.

UFS e CEPED, 2012. Atlas brasileiro de desastres natu- rais 1991 a 2010: volume Brasil. Universidade Federal de Santa Catarina e Centro Universitário de Estudos e Pesquisas sobre Desastres, Florianópolis, Brasil, 94 pp.

Valiente, O.M., 2001. Sequía: definiciones, tipologías y métodos de cuantificación. Investigaciones Geográficas 26: 59-80.

Vaseghi, S.V., 2000. Advanced Digital Signal Processing and Noise Reduction: Fourth edition. John Wiley and Sons, United Kingdom, 544 pp.

Vicente-Serrano, S. M.; S. Beguería, J. Lorenzo-Lacruz, J. Camarero, J. I. López-Moreno, C. Azorin-Molina and A. Sanchez-Lorenzo, 2012. Performance of drought indices for ecological, agricultural, and hydrological applications. Earth Interactions 16(10): 1-27.

Vicente-Serrano, S.M., 2006. Differences in spatial patterns of drought on different time scales: an analysis of the Iberian Peninsula, Water Resource Manage 20(1):37-60.

Wilks, D., 2006. Statistical methods in the atmospheric sciences: second edition. ELSEVIER, Department of Earth and Atmospheric Sciences. Cornell University, USA, 649 pp.

World Water Assessment Programme, 2009. The United Nations World Water Development Report 3: Water in a Changing World. UNESCO, Paris, London, 349 pp.

Yamazaki, Y. and V. Rao, 1977. Tropical cloudiness over South Atlantic Ocean. Journal Meteorological Society Japan 55: 205-207. 